\title{
Collaboration à une initiative de vaccination menée par la santé publique
}

\author{
Desai S. ${ }^{1 *}$, Tsang R. ${ }^{2}$, St. Laurent M. ${ }^{1}$, Cox A. ${ }^{3}$ \\ ${ }^{1}$ Centre de l'immunisation et des maladies respiratoires infectieuses, Agence de la santé publique du Canada, Ottawa (Ontario) \\ ${ }^{2}$ Laboratoire national de microbiologie, Agence de la santé publique du Canada, Winnipeg (Manitoba) \\ ${ }^{3}$ Programme de vaccination, Produits thérapeutiques pour la santé humaine, Conseil national de recherches du Canada, \\ Ottawa (Ontario) \\ * Auteure-ressource : shalini.desai@oahpp.ca
}

\section{Résumé}

La surveillance de la maladie peut être utilisée comme une occasion de déterminer les priorités en matière de recherche et l'élaboration de nouveaux traitements. C'est ce que permettent de constater les travaux en cours visant à mettre au point un nouveau vaccin antigrippal afin de lutter contre une grave maladie d'enfance invasive : l'infection à Haemophilus influenzae de sérotype a (Hia). Après l'introduction du vaccin anti-Hib au calendrier de vaccination systématique des enfants au Canada au début des années 1990, l'Agence de la santé publique du Canada (ASPC) a commencé à documenter la chute des taux d'infection à Haemophilus influenzae de sérotype b (Hib). Cependant, le nombre d'infections invasives attribuables aux souches de $H$. influenzae de type autre que b a commencé à augmenter en 2007, et on a lancé la surveillance des infections invasives attribuables à toutes les souches de $H$. influenzae, ainsi qu'aux souches non typables. Les données les plus récentes semblent indiquer que le Hia est la cause d'une maladie invasive grave, en particulier dans les populations autochtones. De façon semblable au Hib, le Hia cause des maladies graves telles que la méningite, la septicémie et la pneumonie bactériémique chez les jeunes enfants âgés de moins de 5 ans.

Compte tenu de cette nouvelle menace attribuable au Hia chez les populations autochtones au Canada, l'Agence de la santé publique du Canada a noué un partenariat avec le Conseil national de recherches du Canada (CNR) afin d'étudier la possibilité de créer un vaccin polysaccharidique capsulaire contre le Hia. À l'heure actuelle, les souches de semence du vaccin expérimental ont été déterminées, et l'Agence de la santé publique du Canada et le Conseil national de recherches du Canada travaillent de concert avec l'École de médecine du Nord de I'Ontario, les Centers for Disease Control and Prevention des États-Unis et d'autres organismes. Cette recherche a pour but de déterminer et de préparer un vaccin expérimental contre le Hia, tout en améliorant la compréhension de la façon dont un tel vaccin pourrait améliorer la santé d'une population vulnérable.

\section{Introduction}

L'un des avantages de la surveillance de la maladie est qu'elle peut mettre en évidence la nécessité ou l'occasion de nouvelles interventions. La surveillance fournit un des moyens essentiels de détermination des maladies qui créent un fardeau important au sein d'une population et elle aide à déterminer la nécessité d'administrer des vaccins, l'une des interventions en santé publique les plus efficaces pour prévenir l'apparition de la maladie. Par exemple, les vaccins contre la variole ont éradiqué cette maladie (1) et la poliomyélite a été éliminée dans presque tous les pays du monde (2). Après l'obtention des données de surveillance, on a créé des vaccins afin de prévenir les maladies, telles que Streptococcus pneumoniae, Neisseria meningitidis et la grippe.

Dans le cadre de son rôle de servir le bien public, par l'intermédiaire de la surveillance, le secteur de la santé publique peut déterminer la nécessité de nouveaux vaccins contre les maladies qui causent une morbidité et une mortalité importantes au sein de la population, et en faciliter la mise au point.

Le présent article décrit une collaboration axée sur la santé publique qui est apparue entre l'Agence de la santé publique du Canada (ASPC ou l'Agence) et le Conseil national de recherches du Canada (CNR) afin de mettre au point un vaccin qui lutte contre un agent pathogène émergent particulièrement prévalent au sein de la population autochtone : Haemophilus influenzae de sérotype a (Hia). 


\section{Agence de la santé publique du Canada}

L'Agence de la santé publique du Canada a été créée en 2004 pour fournir le leadership fédéral pour prévoir les problèmes de santé publique et les urgences, intervenir et en être responsable (3). L'Agence a également été envisagée comme un moyen d'améliorer la collaboration entre les diverses provinces et les divers territoires pour protéger et promouvoir la santé publique au sein de la population canadienne. Au sein de l'Agence, le Centre de l'immunisation et des maladies respiratoires infectieuses (CIMRI) (4) et le Laboratoire national de microbiologie (LNM) (5) sont les principaux organismes responsables de la prestation de soutien technique pour les maladies évitables par la vaccination. Le CIMRI est responsable de la surveillance épidémiologique de nombreuses maladies évitables par la vaccination, tandis que le LNM offre de la surveillance en laboratoire, des diagnostics de référence et de la recherche. En collaboration, ils fournissent des données sur les nouvelles tendances pour un certain nombre de maladies infectieuses.

\section{Conseil national de recherches du Canada}

Le CNR a commencé son travail afin de mettre au point des technologies de vaccination durant les années 1980. Son domaine d'expertise s'est élargi au fil des ans afin d'inclure l'excellence en chimie des glucides, en immunochimie et en établissement de partenariats afin de créer de nouveaux vaccins $(6,7,8)$. Le CNR a également mis au point une expertise en vue d'améliorer les technologies et les vaccins actuels de façon à poursuivre l'innovation en matière de vaccins. Par l'intermédiaire du CNR, on trouve des services techniques et consultatifs, des établissements de recherche, des possibilités de licence ainsi que des possibilités de programmes et de partenariats. Cette expertise a été utilisée pour étudier les antigènes de glucides d'agents pathogènes bactériens encapsulés tels que Haemophilius influenzae de sérotype b (9), Neisseria meningitidis (6, 7) et Neisseria streptococci (10), qui sont des agents pathogènes importants. Chacun de ces agents pathogènes possède un polysaccharide capsulaire qui contribue à accroître sa virulence. Au cours des dernières années, le CNR s'est concentré sur la sélection de l'antigène, les technologies de glycoconjugaison, la conception d'adjuvants et la production de vaccins expérimentaux tous compatibles avec les bonnes pratiques de fabrication actuelles. Le CNR a également la capacité de mener des essais précliniques in vivo pour effectuer le suivi de l'efficacité et de l'innocuité des produits (11).

\section{Infection invasive à Haemophilus influenzae au Canada}

Avant la mise au point d'un vaccin conjugué anti-H. influenzae de sérotype b (Hib), Hib était une importante cause de la méningite, de la septicémie et de la pneumonie bactériémique chez les enfants âgés de moins de cinq ans. Selon la surveillance à l'échelle nationale de 1986-1987, environ 700 cas d'infection invasive à Hib se sont produits par année. Les vaccins anti-Hib ont été introduits dans le calendrier de vaccination systématique des enfants au Canada au début des années 1990. Depuis l'introduction du vaccin, le taux d'incidence de l'infection à Hib a diminué de façon spectaculaire. Au cours des cinq dernières années pour lesquelles des données sont disponibles à l'échelle nationale (de 2008 à 2012), on a observé une moyenne de 26 cas d'infection à Hib signalés au Canada par année (12). Toutefois, selon les données issues des programmes de surveillance nationale, les infections invasives à $H$. influenzae attribuables à des souches autres que Hib ont émergé au cours de la dernière décennie (13). Par conséquent, en 2007, on a approuvé une révision de la définition nationale de cas d'infection invasive à $H$. influenzae pour inclure les maladies attribuables à tous les sérotypes, ainsi que les souches non typables (14). 
Parmi les souches encapsulées sérotypables de $H$. influenzae, on a déterminé que le sérotype b est le plus virulent suivi par le Hia, puis les autres (15). Les données actuelles révèlent que $H$. influenzae de sérotype a (Hia) est une cause de graves maladies invasives, en particulier dans la population autochtone (16). De façon semblable à Hib, le Hia cause des maladies graves telles que la méningite, la septicémie et la pneumonie bactériémique chez les jeunes enfants âgés de moins de 5 ans. Étant donné que le nord du Canada compte une population autochtone relativement élevée, Hia, qui ressemble à Hib, y compris en matière d'épidémiologie, a remplacé Hib comme cause d'un fardeau important de maladies invasives dans la période suivant le vaccin antiHib (17). Cette tendance a également été observée en Alaska, États-Unis (18).

\section{Collaboration axée sur la santé publique}

Compte tenu de la connaissance d'une nouvelle menace attribuable à Hia chez les populations autochtones au Canada et du lancement d'un programme de vaccination au sein du portefeuille des Produits thérapeutiques pour la santé humaine du CNR, l'ASPC s'est associée au CNR dans le but d'étudier la possibilité de créer un vaccin polysaccharidique capsulaire contre le Hia pour protéger les Canadiens. La première réunion et consultation entre I'ASPC et le CNR a eu lieu en 2011 dans le but de discuter de la faisabilité de la mise au point d'un vaccin glycoconjugé contre le Hia. En octobre 2011, un atelier organisé par l'ASPC, le CNR et l'École de médecine du Nord de l'Ontario a eu lieu à l'Université Lakehead. Cet atelier rassemblait des chercheurs universitaires de l'École de médecine du Nord de l'Ontario et du Thunder Bay Regional Health Authority qui ont discuté de deux maladies infectieuses négligées au sein des communautés autochtones (Hia et Helicobacter pylori) (19). À partir des bases des premières réunions, I'ASPC et le CNR ont signé une lettre d'intention sur l'expansion de la mise au point de vaccins au Canada en septembre 2012, et le projet conjoint sur le Hia a été lancé en mars 2013. En décembre 2013, des chercheurs de l'Arctic Investigators Program des Centers for Disease Control and Prevention (CDC) des États-Unis se sont joints au groupe puisqu'ils avaient également observé une augmentation similaire de l'incidence du Hia au sein de la population autochtone de l'Alaska.

Pour le moment, les souches de semence du vaccin expérimental ont été déterminées et sont en cours de caractérisation par le LNM. Le CNR travaille actuellement à la préparation à grande échelle de glycoconjugats à partir du polysaccharide capsulaire de Hia. Les tests sérologiques, y compris les tests pour mesurer l'immunité protectrice sont actuellement mis au point à l'École de médecine du Nord de l'Ontario. Les études d'immunogénicité chez les animaux qui ont reçu les glycoconjugats sont actuellement effectuées au CNR et le sérum prélevé chez les animaux immunisés est en cours d'évaluation par rapport à un éventail de souches cliniques à l'École de médecine du Nord de l'Ontario.

Cette recherche a pour but de déterminer et de préparer un vaccin expérimental contre le Hia, tout en améliorant la compréhension de la façon dont un tel vaccin pourrait améliorer la santé d'une population vulnérable. Le travail effectué à ce jour était axé sur la façon de fournir une meilleure compréhension et caractérisation de l'épidémiologie de l'infection invasive à Hia grâce à l'amélioration des activités de surveillance. Ce travail s'effectue en collaboration avec des partenaires internationaux tels que les Centers for Disease Control and Prevention et l'Organisation panaméricaine de la santé (OPS). De plus, du côté du laboratoire, ce travail permettra de fournir une validation de concept que le polysaccharide capsulaire de Hia peut être purifié et conjugué à une protéine porteuse qui provoquera une réponse immunitaire qui confère une protection aux animaux de laboratoire. Une fois qu'un vaccin expérimental s'est révélé sûr et efficace dans les essais précliniques, sa production à plus grande échelle nécessite l'engagement de l'industrie ou d'un autre tiers. 


\section{Conclusion}

Ce projet de collaboration est un excellent exemple de la façon dont le leadership gouvernemental peut s'avérer efficace pour répondre à un besoin de santé publique. II montre aussi comment un programme de recherche canadien déterminé peut être élargi et utilisé à l'échelle internationale. Par l'intermédiaire de la surveillance, I'ASPC a la capacité de cerner les possibilités de nouvelles stratégies en matière de vaccination. Le CNR possède l'expertise technique nécessaire à la mise au point et à la production de vaccins expérimentaux. De concert avec d'autres partenaires, comme le milieu universitaire et d'autres organismes gouvernementaux et non gouvernementaux, ce modèle pourrait mener à une autre innovation en matière de vaccination.

\section{Remerciements}

Les auteurs tiennent à remercier John Spika, Jim Richards, Marina Ulanova et Luis Barreto pour leur travail à la mise en place de ce projet de collaboration.

\section{Conflit d'intérêts}

II n'y a aucun conflit d'intérêts à déclarer.

\section{Financement}

Ce travail a été appuyé par l'Agence de la santé publique du Canada et le Conseil national de recherches du Canada.

\section{Références}

(1) Organisation mondiale de la Santé. [en ligne]. Genève, Suisse : Organisation mondiale de la Santé [consulté le 15 septembre 2014]. Accès : http://www.who.int/csr/disease/smallpox/en/

(2) Organisation mondiale de la Santé. [en ligne]. Genève, Suisse : Organisation mondiale de la Santé [mis à jour le 9 septembre 2014; consulté le 15 septembre 2014]. Accès : http://www.polioeradication.org/dataandmonitoring.aspx

(3) Agence de la santé publique du Canada. [en ligne]. Ottawa, Canada : Agence de la santé publique du Canada [mis à jour le 16 juin 2011; consulté le 20 août 2014]. Accès : http://www.phac-aspc.gc.ca/about_apropos/what-fra.php

(4) Agence de la santé publique du Canada. Le Centre de l'immunisation et des maladies respiratoires infectieuses (CIMRI) [en ligne]. Ottawa, Canada : Agence de la santé publique du Canada [mis à jour le 23 juillet 2012; consulté le 20 août 2014]. Accès : http://www.phac-aspc.gc.ca/irid-diir/index-fra.php

(5) Agence de la santé publique du Canada. Laboratoire national de microbiologie [en ligne]. Ottawa, Canada : Agence de la santé publique du Canada [mis à jour le 8 juillet 2014; consulté le 20 août 2014]. Accès : https://www.nmlInm.gc.ca/overview-apercu-fra.htm

(6) Jennings HJ, Lugowski C, Ashton FE. Conjugation of meningococcal lipopolysaccharide R-type oligosaccharides to tetanus toxoid as route to a potential vaccine against group B Neisseria meningitidis. Infect Immun. 1984 Jan; 43(1):407-12.

(7) Jennings HJ, Lugowski C. Immunochemistry of groups A, B, and C meningococcal polysaccharide-tetanus toxoid conjugates. J Immunol. $1981 \mathrm{Sep}$; 127(3):1011-8.

(8) Jennings HJ, Lugowski C, inventors; National Research Council of Canada, assignee. Immunogenic polysaccharideprotein conjugates. United States patent US4356170A. 1982 Oct 26.

(9) Jennings HJ. Capsular polysaccharides as human vaccines. Adv Carbohydr Chem Biochem. 1983; 41:155-208.

(10) Kasper DL, Paoletti LC, Wessels MR, Guttormsen HK, Carey VJ, Jennings HJ, Baker CJ. Immune response to type III group B streptococcal polysaccharide-tetanus toxoid conjugate vaccine. J Clin Invest. 1996 Nov 15; 98(10):2308-14.

(11) Conseil national de recherches Canada. À propos du CNRC [en ligne]. Ottawa, Canada : Conseil national de recherches Canada [mis à jour le 15 juillet 2014; consulté le 20 août 2014]. Accès : http://www.nrccnrc.gc.ca/fra/apropos/index.html

(12) Agence de la santé publique du Canada. Maladies à déclaration obligatoire en direct. [en ligne] Ottawa, Canada : Agence de la santé publique du Canada. [mis à jour le 31 mars 2014; consulté le 3 août 2014] Accès : http://dsolsmed.phac-aspc.gc.ca/dsol-smed/ndis/graphiques.php?c=pl 
(13) Tsang R. Changing epidemiology of invasive Haemophilus influenzae disease. Lancet Infectious Diseases, 2008; 8:737.

(14) Agence de la santé publique du Canada. Case definitions for communicable diseases under national surveillance. Can Commun Dis Rep. 2014 Août 20; 35(Suppl. 2):1-40.

(15) Zwahlen A, Kroll JS, Rubin LG, Moxon R. The molecular basis of pathogenicity in Haemophilus influenzae: comparative virulence of genetically related capsular transformants and correlation with changes of the capsulation locus cap. Microbial Pathogenesis. 1989; 7:225-235.

(16) Ulanova M, Tsang RSW. Haemophilus influenzae serotype a as a cause of serious invasive infections. The Lancet Infectious Diseases. 2004; 14:70-82.

(17) Rotondo J, Sherrard L, Helferty M, Tsang R, Desai S. The epidemiology of invasive disease due to Haemophilus influenza serotype a in the Canadian North from 2000 to 2010. Int J circumpolar Health. 2013; 72:1-5.

(18) Bruce MG, Zulz T, DeByle C, Singleton R, Hurlburt D, Bruden D, Rudolph K, Hennessy T, Klejka J, Wenger JD. Haemophilus influenzae serotype a invasive disease, Alaska, USA, 1983-2011. Emerg Infect Dis. 2013 Jun; 19(6):9327.

(19) Ulanova M, Tsang R, Altman E. Neglected infectious diseases in Aboriginal communities: Haemophilus influenzae serotype a and Helicobacter pylori. Vaccine. 2012 Nov 19; 30(49):6960-6. 